\title{
Colorimetric Sensing of Cysteine in serum by the competitive adsorption toward AuNPs between Cysteine and ATP
}

\author{
Lingzhi Zhao ${ }^{1,3, a^{*}}$, Liu Zhao ${ }^{2, b}$, Yanqing Miao ${ }^{1, c}$, Chunye Liu ${ }^{1, d}$ \\ ${ }^{1}$ Department of pharmacy, Xi'an Medical College, Xi'an 710021, China \\ ${ }^{2}$ Beijing Research Center of Agricultural Standards and Testing, Beijing 100097, China \\ ${ }^{3}$ Key Laboratory of Analytical Chemistry for Life Science of Shaanxi Province, School of Chemistry \\ and Chemical Engineering, Shaanxi Normal University, Xi'an 710062, China \\ a oldskyhappy_zlz@163.com
}

\begin{abstract}
Keywords: colorimetric; cysteine; reduced glutathione; homocysteine; Gold nanoparticles (AuNPs) Abstract: Based on the dependence between the AuNPs size and the naked-eye visible color change. This study was designed to develop a highly selective and sensitive towards colorimetric sensing of cysteine in fetal bovine serum by the competitive binding of ATP and thiol compounds (cysteine and homocysteine) towards gold nanoparticles (AuNPs). The as-established method has high sensitivity and selectivity, the linearity range was $3 \mu \mathrm{M}-30 \mu \mathrm{M}$ with the quantification limit of $1 \mu \mathrm{M}$. The method demonstrated here can be applied to detect cysteine in fetal bovine serum without interference from other amino acids and bovine serum albumin (BSA), which is envisaged to be applicable to understanding the chemical essence involved in physiological and pathological events associated with cysteine.
\end{abstract}

\section{Introduction}

As one kind of thiol-containing amino acid, cysteine deficiency is involved in many syndromes, such as slower growth, liver damage, edema, skin lesions and so forth. Thus, it is very important to develop some simple analytical detection methods for monitoring total plasma cysteine levels ${ }^{[1]}$. The majority of the reported methods for analysis of cysteine are electrochemical methods based on its redox chemistry, fluorescent probe technology based on the strong nucleophilicity of a thiol group, or capillary electrophoresis with mass spectrometric and so forth ${ }^{[2-4]}$. These methods have their own drawbacks and advantages. Herein, we demonstrate a novel method for the selective detection of micromolar concentrations of cysteine in fetal bovine serum with Au nanoparticles (AuNPs) as probes.

AuNPs, with their excellent biocompatibility and size-dependent surface plasmon resonance (SPR) absorption, have been employed as a colorimetric probe in a variety of research fields, such as the measurement of proteins, metal ions and other small biomolecules, the analysis of the enzymatic activity and the screening of enzyme inhibitors, the analysis of DNA hybridization and so forth ${ }^{[5-10]}$. In this study, our detection principle is based on competitive adsorption between cysteine and ATP toward AuNPs. In details, cysteine replaced ATP molecules owing to Au-S bond formation and lead to the aggregation of Au-NPs without the protection of ATP at high salt concentration. The as-established method can be applied to detect spiked cysteine in fetal bovine serum with good recovery without interference from other amino acids (containing reduced glutathione, GSH) and BSA except homocysteine. Colorimetric sensing of cysteine with AuNPs as probes, since the as-established methods only contain very simple sample handling procedures and low instrumental investments, are quite promising to be developed as portable devices for understanding the chemical essence involved in physiological and pathological events associated with cysteine. 


\section{Chemicals and Reagents}

Chloroauric acid $\left(\mathrm{HAuCl}_{4} \cdot 3 \mathrm{H}_{2} \mathrm{O}\right)$, trisodium citrate, L-Cysteine, Adenosine 5-triphosphate disodium salt (ATP), Other amino acids were purchased from Aladdin Company. Homocysteine and reduced glutathione were purchased from Sigma-Aldrich, fetal bovine serum was purchased from Hyclone. Aqueous solutions of GSH, cystine and homocysteine were freshly prepared with PBS prior to experiments. Phosphate-buffered saline (PBS, $\mathrm{pH} 7.4$ ) was prepared by mixing $136.7 \mathrm{mM} \mathrm{NaCl}$, $2.7 \mathrm{mM} \mathrm{KCl}, 0.087 \mathrm{M} \mathrm{Na}_{2} \mathrm{HPO}_{4}$, and $0.014 \mathrm{M} \mathrm{KH}_{2} \mathrm{PO}_{4}$ into water. All reagents were at least analytical grade and used as received. Aqueous solutions were prepared with Milli-Q water.

\section{Synthesis of AuNPs}

Au-NPs with a diameter of about $13.5 \mathrm{~nm}$ were synthesized as reported before. Briefly, trisodium citrate $(10 \mathrm{~mL}, 38.8 \mathrm{mM})$ was added to a boiling solution of $\mathrm{HAuCl}_{4}(100 \mathrm{~mL}, 1 \mathrm{mM})$, and the resulting solution was kept continuously boiling for another $30 \mathrm{~min}$ to give a wine red mixture. The mixture was cooled to room temperature and then filtrated through a Millipore syringe $(0.45 \mu \mathrm{m})$ to remove the precipitate, and the filtrate was stored in a refrigerator at $4{ }^{\circ} \mathrm{C}$ for use. UV-vis spectroscopy (UV 2012-PCS spectrophotometer, Unico (Shanghai) Instrument Co. Ltd., China) with an absorption peak at around $520 \mathrm{~nm}$.

\section{Colorimetric Sensing of Cysteine in fetal bovine serum}

The samples were all prepared by dissolving cysteine directly into PBS as standard cysteine solution. $20 \mu \mathrm{L}$ standard cysteine solution with different concentration and $400 \mu \mathrm{L}$ methanol were added to $180 \mu \mathrm{L}$ of serum and vortexed thoroughly. The mixture was allowed to stand for about $10 \mathrm{~min}$ at room temperature to complete protein precipitation. After centrifugation at $13,000 \times \mathrm{g}$ in an Eppendorf centrifuge, the solvent was evaporated to dry and samples were redissolved in $20 \mu \mathrm{L} \mathrm{PBS.}$ For the colorimetric sensing of cysteine in fetal bovine serum, $20 \mu \mathrm{L}$ samples was first added into $240 \mu \mathrm{L}$ of the aqueous dispersion of Au-NPs containing ATP $(20 \mu \mathrm{L}, 1 \mathrm{mM})$ and $\mathrm{NaCl}(20 \mu \mathrm{L}, 30 \mathrm{mM})$, After being allowed to stand by for 40min, the mixtures were photographed, and the concentrations of cysteine in fetal bovine serum were determined with UV-vis spectroscopy.

\section{Results and Discussion}

\section{Mechanistic Investigation on Colorimetric Cysteine Sensing}

Scheme 1 illustrates the method for the detection of cysteine, AuNPs synthesized with citrate as the stabilizer were red in color and exhibit absorption at $520 \mathrm{~nm}$. In the absence of ATP, the sole addition of $30 \mathrm{mM} \mathrm{NaCl}$ lead to an observable aggregation of AuNPs with color changes from red to blue. In the presence of $1 \mathrm{mM}$ ATP, through the coordinating interaction of Adenosine and AuNPs, AuNPs can be stabilized owing to the negatively charged phosphate group which prevented the AuNPs from aggregating through electrostatic repulsion without color change. Upon the addition of cysteine, the ATP-protected AuNPs had an observable aggregation accompanying clear color changes from red to blue due to the competitive adsorption between cysteine and ATP. The result indicates that the ATP molecules were replaced by cysteine owing to $\mathrm{Au}-\mathrm{S}$ bond formation and released from the surface of AuNPs, confirming the stronger binding ability of cysteine and Au-NPs, Au-NPs lose the protection of ATP, $30 \mathrm{mM} \mathrm{NaCl}$ result in the aggregation of Au-NPs with color changes from red to blue once again. These features substantially constitute a straight forward basis for colorimetric sensing of cysteine through the changes in the color and UV-vis spectra of the Au-NPs dispersion. 


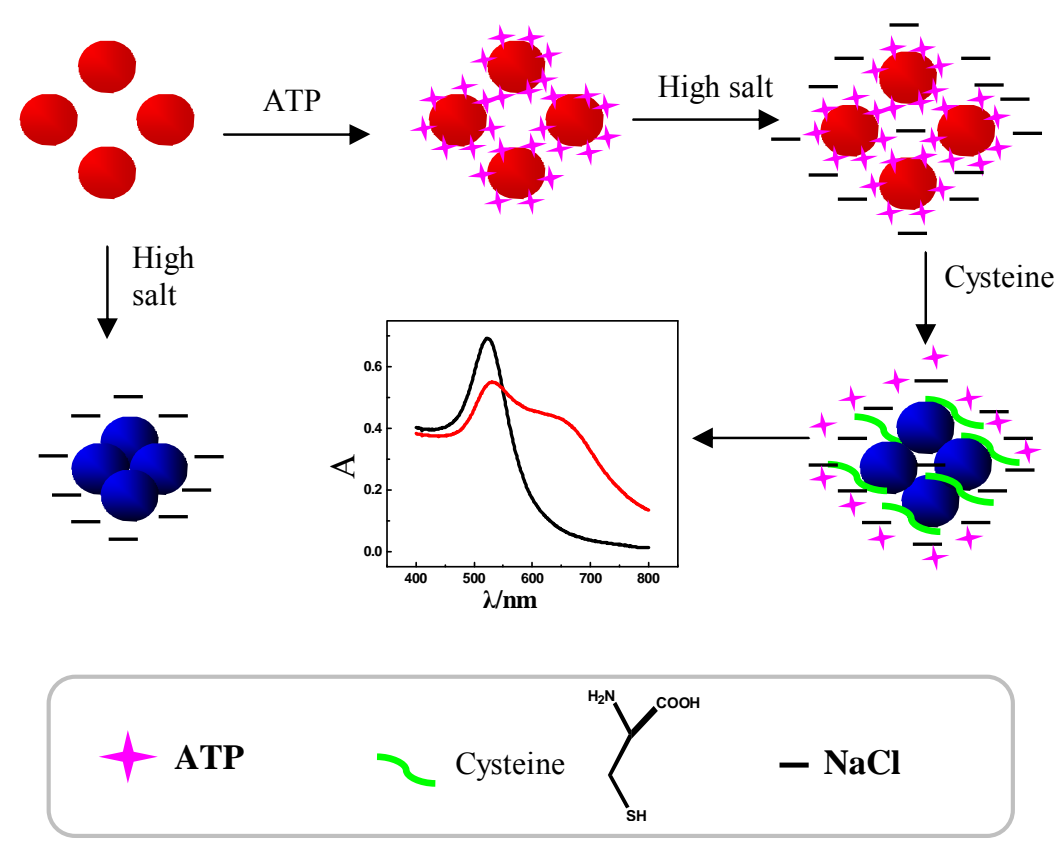

Scheme 1. Schematic illustration of the change in the dispersion/aggregation states of AuNPs evoked by the competitive coordination chemistry between cysteine and ATP for colorimetric sensing of cysteine.

\section{Optimal conditions}

The cysteine-induced gold nanoparticle aggregation and ATP-triggered disassembly kinetics were evaluated in Fig.1. A change in UV-vis spectra of AuNPs after the addition of cysteine $(10 \mu \mathrm{M})$ was observed, there is a gradual increase in absorption peak around $650 \mathrm{~nm}$ with time, It is assumed that the competitive coordination chemistry between cysteine and ATP have achieved a balance and that no further aggregation of AuNPs occurred. Therefore, the interaction time required for optimum color change of AuNPs in the presence of cysteine was taken after $40 \mathrm{~min}$.

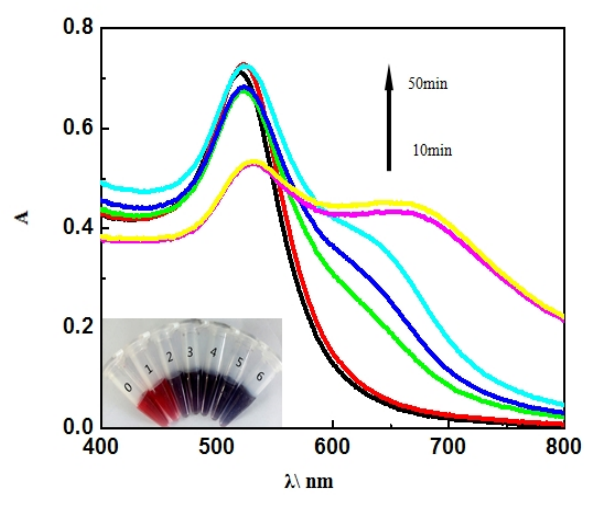

Fig.1 UV-vis spectra and photographs (left corner) for the effect of time on the cysteine-triggered assembly of the ATP-protected AuNPs. Black, bare AuNPs; red, AuNPs containing ATP $(20 \mu \mathrm{L}, 1 \mathrm{mM})$ and $\mathrm{NaCl}(20 \mu \mathrm{L}, 30 \mathrm{mM})$; AuNPs containing $1 \mathrm{mM} \mathrm{ATP}$ and $30 \mathrm{mM} \mathrm{NaCl}$ incubating $10 \mu \mathrm{M}$ Cysteine for different time 10 (green), 20 (blue), 30 (cyan), 40 (magenta), 50min (yellow). Concentration: AuNPs, 5nM.

\section{Selectivity and Sensitivity}

To evaluate the sensitivity of our method, in a first try, we added other amino acids and BSA with the same concentrations $10 \mu \mathrm{M}$ into the dispersive ATP-protected AuNPs, as depicted in Figure 2A, except cysteine and homocysteine, the addition of other amino acids and BSA did not result in the 
Au-NPs aggregation. These results substantially demonstrate that the present method has a high selectivity against these amino acids and BSA.

Because homocysteine also caused an observable Au-NPs aggregation as cysteine, we next studied the selectivity of the colorimetric method for sensing cysteine among the thiol-containing analogues such as homocysteine and glutathione due to their high structural similarity to cysteine. As shown as Fig.3, different concentrations of homocysteine in PBS were added into the aqueous dispersion of ATP-protected Au-NPs to cause the aggregation of AuNPs in color (from red to purple-blue) and in the UV -vis spectrum. These changes eventually constitute a straightforward basis for colorimetric sensing of homocysteine. The ratio of $\left(\mathrm{A}_{650} / \mathrm{A}_{520}\right.$ increases with homocysteine concentration and shows a linear response toward homocysteine within a concentration range from $5 \mu \mathrm{M}$ to $50 \mu \mathrm{M}\left(\mathrm{A}_{650} / \mathrm{A}_{520}=0.0315 \mathrm{C} / \mu \mathrm{M}+0.2686, \mathrm{R}^{2}=0.9752\right)$. Moreover, different from homocysteine, GSH in PBS were added into the aqueous dispersion of ATP-protected Au-NPs to cause no evident change of color and UV-vis spectrum, which suggesting no interference from GSH for sensing cysteine.
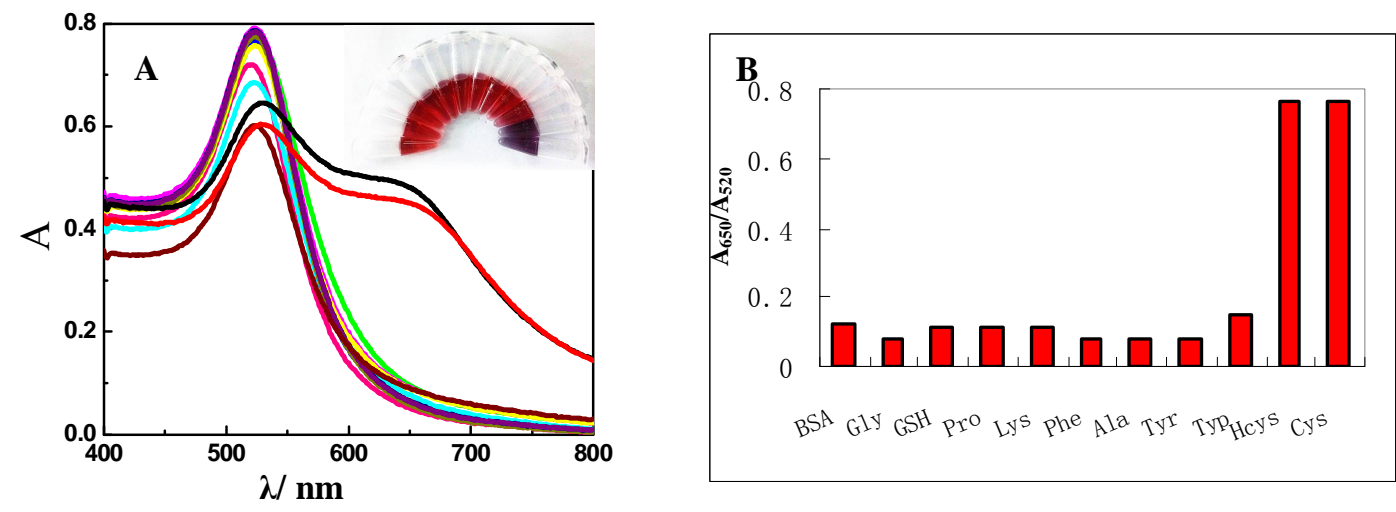

Fig.2 (A) UV-vis spectra and photographs (inset) of aqueous dispersions of AuNPs containing ATP $(20 \mu \mathrm{L}, 1 \mathrm{mM})$ and $\mathrm{NaCl}(20 \mu \mathrm{L}, 30 \mathrm{mM})$ with the addition of different compounds. (B) Values of $\mathrm{A}_{650} / \mathrm{A}_{520}$ containing ATP $(20 \mu \mathrm{L}, 1 \mathrm{mM})$ and $\mathrm{NaCl}(20 \mu \mathrm{L}, 30 \mathrm{mM})$ with the presence of various compounds (The final concentration of these compounds in the resulting AuNPs dispersions $(240 \mu \mathrm{L})$ was $10 \mu \mathrm{M}$.
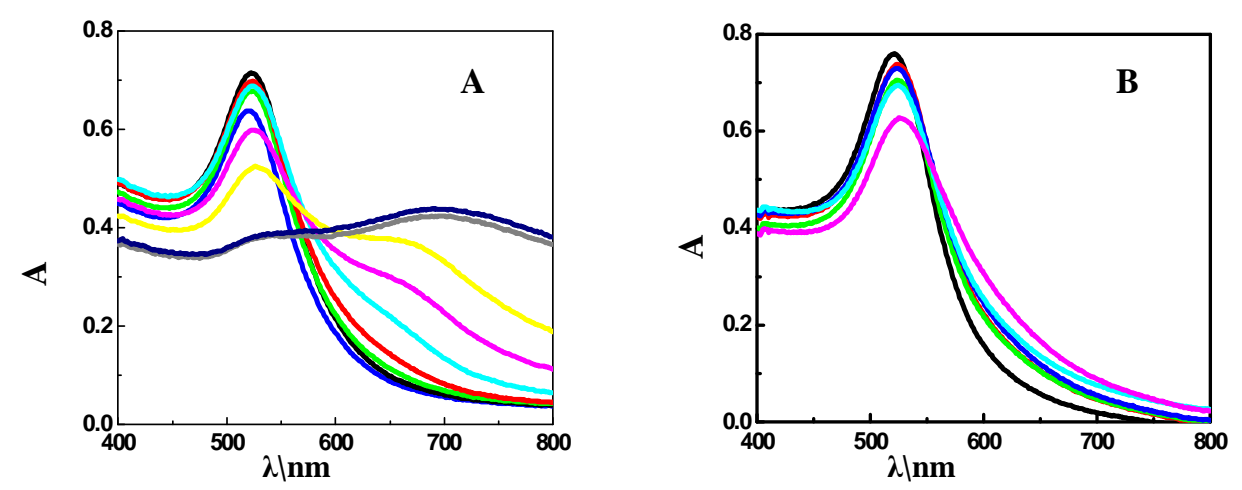

Fig.3 UV-vis spectra of AuNPs prepared by the addition of various concentrations of homocysteine (A) and glutathione (B) to AuNP dispersions containing ATP $(20 \mu \mathrm{L}, 1 \mathrm{mM})$ and $\mathrm{NaCl}$ $(20 \mu \mathrm{L}, 30 \mathrm{mM})$;. Black, bare AuNPs; red, AuNPs containing 1mM ATP and 30mM NaCl; AuNPs containing $1 \mathrm{mM}$ ATP , 30mM NaCl and different concentration of (A) homocysteine 1 (green), 3 (blue), 5(cyan), 10 (magenta), 30(yellow), $50 \mu \mathrm{M}$ (Gray). The different concentration of (B)glutathione in the resulting Au-NP dispersions $(240 \mu \mathrm{L}$ ) were 1(blue), 10 (green), 100 (cyan), $1000 \mu \mathrm{M}$ (magenta).

To evaluate the sensitivity of the assay, different concentrations of cysteine in PBS were added into the aqueous dispersion of ATP-protected AuNPs to cause the aggregation of AuNPs in color (from red 
to purple-blue) and in the UV-vis spectrum in Fig.4. These changes eventually constitute a straightforward basis for colorimetric sensing of cysteine. Under the optimal conditions, The calibration curve were drawn by plotting the absorbance ratio $\mathrm{A}_{650} / \mathrm{A}_{520}$ against the cysteine concentration, which shows a linear response toward cysteine in AuNPs dispersions over a concentration range of $3 \mu \mathrm{M}-30 \mu \mathrm{M}\left(\mathrm{A}_{650} / \mathrm{A}_{520}=0.0582 \mathrm{C} / \mu \mathrm{M}+0.0936, \mathrm{R}^{2}=0.9846\right)$. The detection limit was $200 \mathrm{nM}$, calculated from $\mathrm{S} / \mathrm{N}=3$.
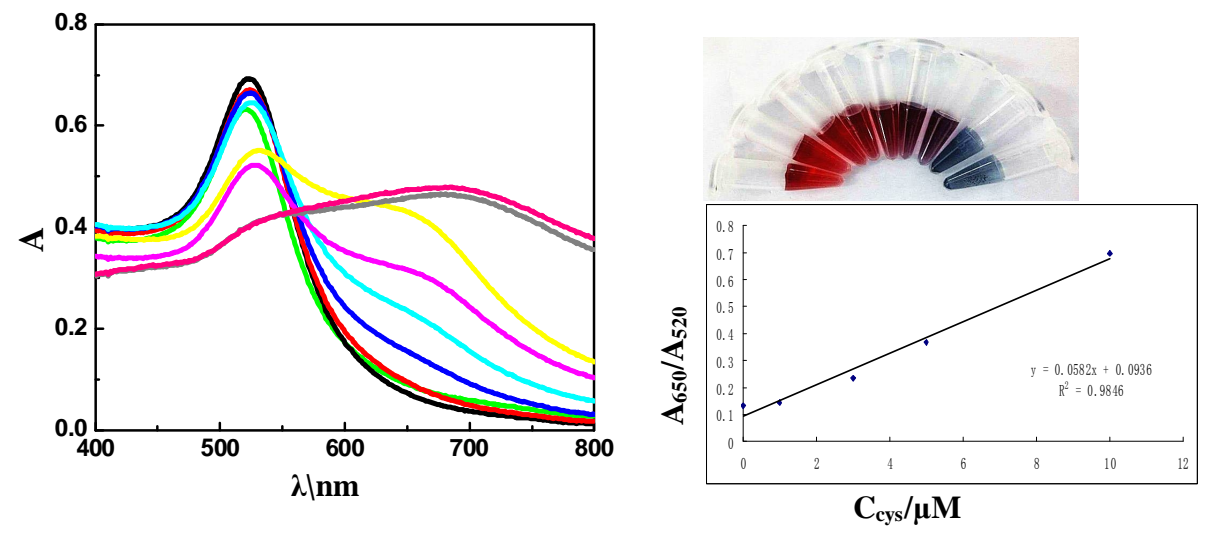

Fig.4 (a) UV-vis spectra and (b) Photographs of Au-NP dispersions prepared by the addition of various concentrations of cysteine to Au-NP dispersions containing ATP $(20 \mu \mathrm{L}, 1 \mathrm{mM})$ and $\mathrm{NaCl}$ $(20 \mu \mathrm{L}, 30 \mathrm{mM}) ;$. The final concentrations of cysteine in the resulting Au-NP dispersions $(240 \mu \mathrm{L})$ were 1 (green), 3(blue), 5(cyan), 10(magenta), 30(yellow), 50 $\mu \mathrm{M}$ (Gray), $80 \mu \mathrm{M}$ (Pink). Right corner: Plot of A650/A520 against $\mathrm{C}_{\text {Cys. }}$.

\section{Colorimetric Sensing of Cysteine in fetal bovine serum}

To demonstrate the suitability of our colorimetric method for cysteine sensing in fetal bovine serum, the serum after protein precipitation treatment was added into the aqueous dispersion of Au-NPs containing $1 \mathrm{mM}$ ATP and $30 \mathrm{mM} \mathrm{NaCl}$, As depicted in Fig. 5, the addition of the purified serum leads to a slight change in the color of the Au-NPs dispersion from red(viall) to somewhat purple (vial 2), and to a clear increase in the $A_{650} / A_{520}$ value in the UV-vis spectra, suggesting the existence of cysteine in fetal bovine serum. To verify that the aggregation of AuNPs was induced by cysteine, the sensitivities of cysteine $(5 \mu \mathrm{M}, 10 \mu \mathrm{M}, 15 \mu \mathrm{M})$ in spiked serum samples were investigated in Fig.5, The results showed that the recovery was above $90 \%$ according to the calibration curve described above. These properties substantially demonstrate that the colorimetric assay developed in this study by exploring the competitive adsorption toward AuNPs between cysteine and ATP would offer an effective way to directly monitoring cysteine in fetal bovine serum.

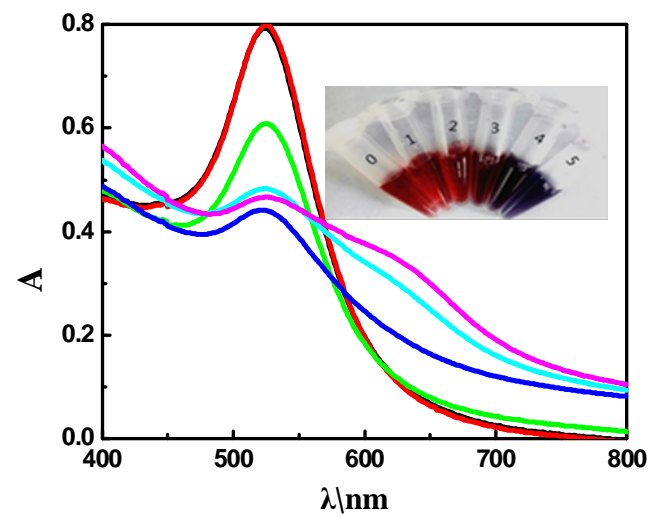

Fig.5. UV-vis spectra and photographs (right corner) of Au-NP dispersions (5nM, 200 $\mu \mathrm{L})$ with the additions of different solutions. Black curve and vial 0: with no addition; red curve and vial 1: with addition of ATP $(20 \mu \mathrm{L}, 1 \mathrm{mM})$ and $\mathrm{NaCl}(20 \mu \mathrm{L}, 30 \mathrm{mM})$; green curve and vial 2: with addition of ATP 
$(20 \mu \mathrm{L}, 1 \mathrm{mM})$ and $\mathrm{NaCl}(20 \mu \mathrm{L}, 30 \mathrm{mM})$ and $20 \mu \mathrm{L}$ serum sample; blue, cyan, magenta curve and vial 3,4,5: with addition of ATP $(20 \mu \mathrm{L}, 1 \mathrm{mM})$ and $\mathrm{NaCl}(20 \mu \mathrm{L}, 30 \mathrm{mM})$ and $20 \mu \mathrm{L}$ spiked serum sample containing $5 \mu \mathrm{M}, 10 \mu \mathrm{M}, 15 \mu \mathrm{M}$ Cysteine.

\section{Conclusions}

In summary, we have developed a simple, sensitive and selective colorimetric sensor for the quantitative detection of cysteine at physiological $\mathrm{pH}$ conditions based on cysteine-induced aggregation of AuNPs in the ATP-AuNPs system. This sensor has high sensitivity and selectivity, the linearity range was $3 \mu \mathrm{M}-30 \mu \mathrm{M}$ with the quantification limit of $1 \mu \mathrm{M}$. The method demonstrated here can be applied to detect cysteine in fetal bovine serum without interference from other amino acids and bovine This colorimetric sensing method provides a general platform for sensing species based on the aggregation of AuNPs transferred from the competition replacement between target analytes and the anti-aggregation agent, which is envisaged to be applicable to understanding the chemical essence involved in physiological and pathological events associated with cysteine.

\section{Acknowledgements}

This work is financially supported by NSF of China (Grant Nos. 21305109 for L. Zhao, 81202492 for C. Liu), by the PhD Startup Foundation of Xi' an Medical University of China (No. 2012DOC09) and by the Scientific Research Plan Projects Foundation of Shaanxi Science and Technology Department of China (No. 2014JQ2073)

\section{References}

[1] G.Y.Mi, D.P.Jones. Cysteine/cystine redox signaling in cardiovascular disease, Free Radical Biology and Medicine. 50 (2011)495-509.

[2] Z.Erhan, et al. An electrochemical and computational study for discrimination of d-and l-cystine by reduced graphene oxide/ $\beta$-cyclodextrin. Analyst. 140 (2015) 313-321.

[3] D.Xi, et al. A simple and effective coumarin-based fluorescent probe for cysteine. Biosensors and Bioelectronics. 59 (2014) 35-39.

[4] R.Chand, et al. Microchip Capillary Electrophoresis Based Separation and Detection of Cysteine and Homocysteine. Advanced Materials Research. 647(2013) 482-486.

[5] V.Diana, M.C.González, A. Escarpa. Sensing colorimetric approaches based on gold and silver nanoparticles aggregation: chemical creativity behind the assay. Analytica chimica acta. 751 (2012) 24-43.

[6] J.J Deng, et al. Competitive coordination of $\mathrm{Cu}^{2+}$ between cysteine and pyrophosphate ion: toward sensitive and selective sensing of pyrophosphate ion in synovial fluid of arthritis patients." Analytical chemistry 85 (2013) 2516-2522.

[7] B.Kong, et al. Sensitive and selective colorimetric visualization of cerebral dopamine based on double molecular recognition. Angewandte Chemie 123 (2011) 1877-1880.

[8] D.S.Seferos, et al. Nano-flares: probes for transfection and mRNA detection in living cells. Journal of the American Chemical Society. 129 (2007) 15477-15479.

[9] Z.Wu, et al. Activity-based DNA-gold nanoparticle probe as colorimetric biosensor for DNA methyltransferase/glycosylase assay. Analytical chemistry 85 (2013) 4376-4383.

[10] E.Hutter, M.Dusica, Gold-nanoparticle-based biosensors for detection of enzyme activity. Trends in pharmacological sciences 34 (2013) 497-507. 